\title{
DESIGN AND PERFORMANCE STUDY OF A UNIQUE MODULAR VERTICAL AXIS WIND TURBINE
}

\author{
YONGJIAN GU ${ }^{1}$, EHSAN KAMEL ${ }^{2}$, ILYA TSITRON ${ }^{3} \&$ MAX AGINSKIY $^{3}$ \\ ${ }^{1}$ United States Merchant Marine Academy, USA \\ ${ }^{2} \mathrm{New}$ York Institute of Technology, USA \\ ${ }^{3}$ Green Power Tower Energy LLC, USA
}

\begin{abstract}
Wind is a green, renewable, and sustainable energy resource. A wind turbine is a device to convert wind energy to electrical energy. There are two kinds of wind turbines: horizontal axis wind turbines (HAWT) and vertical axis wind turbines (VAWT). Compared to the HAWT, the VAWT does not have a tall tower and giant turbine blades. Consequently, the VAWT does not need a large open area as the HAWT does. The VAWT application is attractive in residential and metropolitan areas. This paper presents the design and performance study of a unique modular VAWT, green power tower (GPT). This unique modular VAWT has a cylindrical shape which is able to generate electricity from $360^{\circ}$ wind flow. Turbine blades and shaft of the GPT are entirely covered in a round enclosure. No moving components are exposed to outside. The operation of the GPT is quiet, safe, and environment-friendly. In the paper, the working principle of the GPT is illustrated. In operation, wind stream alternates the flow direction in the enclosure by the redirecting bottom wall and blows up toward the turbine blades vertically. In addition, the modular and packable characteristics of the GPT make its installation convenient to meet the required power generation capacity by using multiple levels. In the paper, the study of the operation performance of the GPT conducted by the scale prototypes is presented. The performance parameters with various wind speeds, such as tip speed ratio $(T S R)$, power coefficient $\left(C_{p}\right)$, and power generation are shown and compared. The comparison of the GPT to the HAWT in equivalent small-scale prototypes was conducted as well. The results showed the GPT is competitive to the HAWT in performance. The manufacturing and installation of the GPT are economically justified.

Keywords: wind energy, wind turbine, vertical axial wind turbine (VAWT), green power tower (GPT).
\end{abstract}

\section{INTRODUCTION}

Wind is a green, renewable, and sustainable energy resource. A wind turbine is a device to extract wind energy and convert it into mechanical energy which in turn is converted into electrical energy. Currently, there are two types of wind turbines that harvest the flow of wind: horizontal axis wind turbine (HAWT) and vertical axis wind turbine (VAWT). Even though the HAWT can have a higher efficiency than traditional VAWT, the HAWT will need a huge structure (tower and blades) and a large open area. Mounting and constructing such large components is expensive and maintaining them is difficult as well. Therefore, a unique modular and stackable VAWT called green power tower (GPT) is proposed as shown in Figs 1 and 2.

The GPT is an omnidirectional wind turbine with no moving part on the outside. Extensive performance research with various experimental tests was performed at New York Institute of Technology (NYIT) through the scale prototypes of the GPT. The preliminary study found that this unique GPT could generate more power compared to the existing VAWT types, such as Savonius, Darreieus, and Giromill, that is, power higher by about $10 \%$.

In light of the modular and stackable characteristics which the GPT has, manufacturing and installation of the GPT are quick and convenient. Each level of the structure is manufactured off-site with a small number of components that just needs to be replicated. The assembly of the GPT takes place on site and the required capacity of the GPT can be met 
8-level GPT
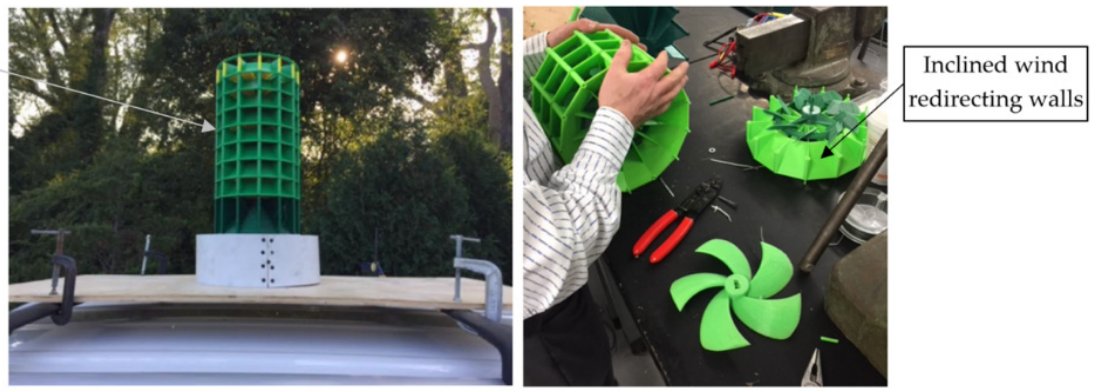

Figure 1: Omnidirectional GPT with no moving part on the outside.

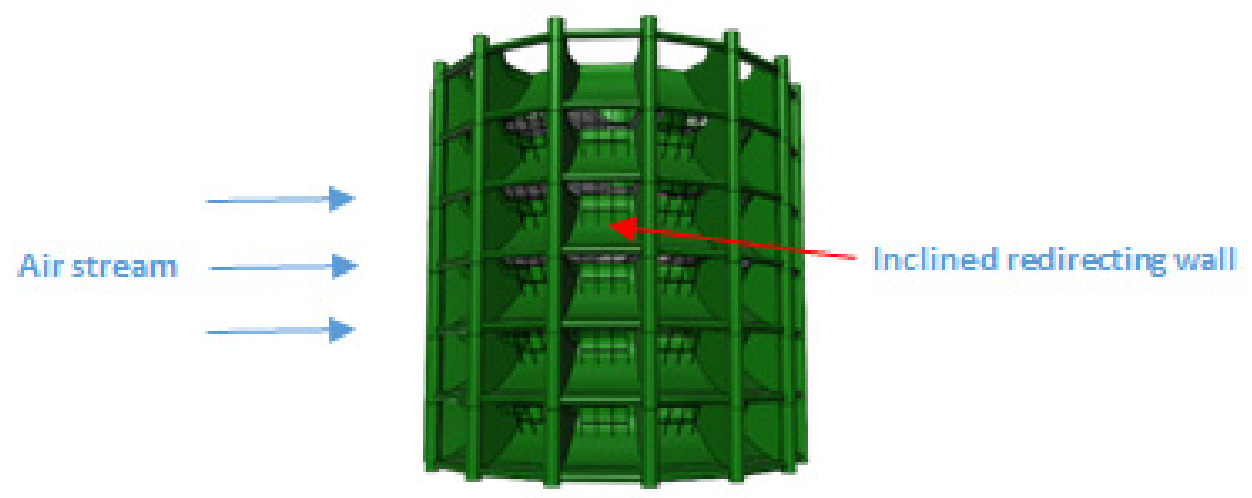

Figure 2: Convert flow from horizontal to vertical in enclosure.

by multilevels. As a result, the cost of the GPT is relatively low compared to that of the conventional wind turbines.

\section{WORKING PRINCIPLE}

As shown in Fig. 2, air stream initially approaches the GPT horizontally. The unique structure of the GPT redirects the wind upward. In the enclosure, the air steam flows along the redirecting bottom wall up to rotate the turbine blades nearly in perpendicular direction. The air finally exhausts out on the top of the GPT. It is an omnidirectional wind turbine technology to generate power by wind from the direction of $360^{\circ}$. In the GPT, all turbine rotating components are covered in the enclosures and not exposed to surroundings. The turbine drives the shaft at the enclosure center. The shaft is connected to the generator located at the bottom (ground) to generate electricity out. One level of the GPT is composed of one enclosure and one turbine. The GPT can be stacked up in multiple levels to meet the designed power load. The manufacturing and installation of the GPT are economically justified. The operation of the GPT is safe and environment friendly.

\section{DESIGN AND PERFORMANCE STUDY}

The design and performance study of the GPT in this paper was based on scale prototypes prior to building real units. Scale HAWT prototypes are also used for a comparison study. 


\subsection{Design of scale prototypes}

The power generation capacity of a wind turbine is proportional to its swept area. The swept area is a circle with a radius equal to the blade's length. In the GPT, the total swept area is equal to the available area in one level multiplied by the number of levels. In the study, the GPT with one, two, and three levels are developed to see the results for power generation, tip speed ratio (TSR), and power coefficient $C_{p}$.

Available power $P_{\text {in }}(k W)$ from wind is calculated by

$$
P_{\text {in }}=\frac{1}{2} A \times \rho_{\text {air }} \times v^{3} \text {, }
$$

where

$\rho_{\text {air }}=$ air density $\left(\mathrm{kg} / \mathrm{m}^{3}\right)$,

$v=$ wind speed $(\mathrm{m} / \mathrm{s})$,

$A=$ swept area $\left(\mathrm{m}^{2}\right)$.

To evaluate the impact of propeller types, three types of turbine blades were considered in the design as shown in Fig. 3. These propeller types were applied in the GPT performance tests. From an aerodynamic view, it does not mean that the more the blades, the more the energy which can be attracted from the blowing air. More blades also will cost more in construction. There should be an optimum blade number based on aerodynamics and economic analysis. Regarding the propeller diameters of the GPT, the limitation was the wind tunnel size in the test. The parameters of both GPT and scale HAWT prototypes used in the experimental study are shown in Table 1.

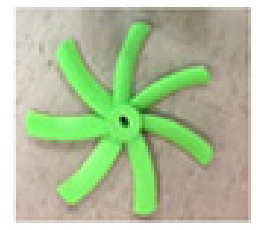

Type A

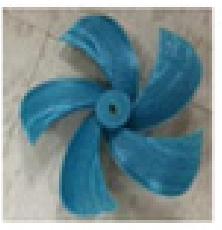

Type B

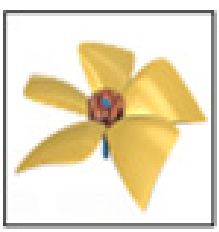

Type C

Figure 3: Propeller types.

Table 1: Parameters of small-scale prototypes of GPT and HAWT.

\begin{tabular}{|l|c|c|}
\hline \multicolumn{1}{|c|}{ Prototype } & $\begin{array}{c}\text { Radius (length of } \\
\text { blade })(\mathrm{cm})\end{array}$ & $\begin{array}{c}\text { Swept Area } \\
\left(\mathrm{cm}^{2}\right)\end{array}$ \\
\hline GPT-One level & 9 & 180 \\
\hline GPT-Two levels & 9 & 270 \\
\hline GPT-Three levels & 9 & 360 \\
\hline GPT-Horizontal propeller & 9 & 255 \\
\hline HAWT-1 & 12 & 450 \\
\hline HAWT-2 & 13 & 530 \\
\hline
\end{tabular}

The GPT prototypes and assembly shown in Fig. 4 were 3D-printed by using PETG (polyethylene terephthalate glycol). 

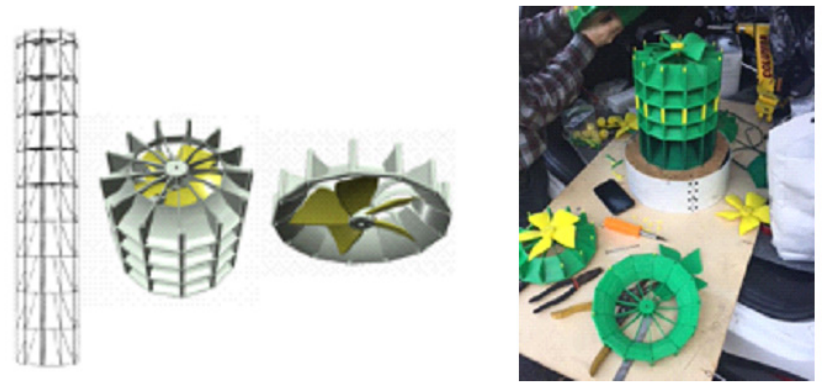

Figure 4: 3D printed modular of GPT prototype.

\subsection{Test equipment and procedure}

The tests were conducted in the aerodynamic lab of NYIT. The major test equipment and devices included the wind tunnel, the load box, the generator, the tachometer and multimeter, and the control panel which was for adjusting the wind speed. Fig. 5 presents the test equipment and devices. The generator was attached to the bottom of GPT and was connected to the load box.

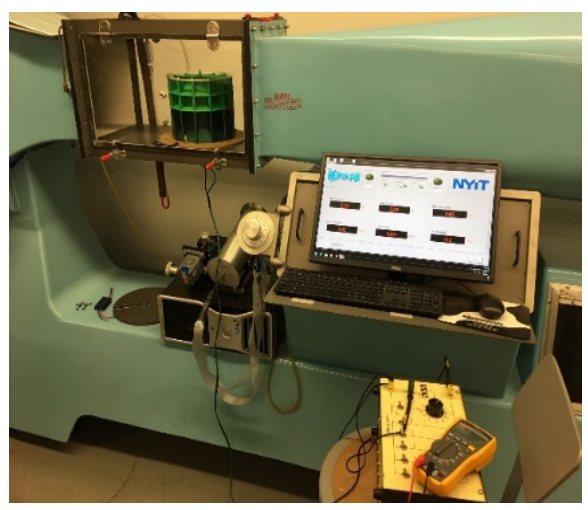

Figure 5: Test equipment and devices.

Depending on the electric resistance and output voltage shown on the load box, the output power could be determined.

The test began with increasing the wind speed in small increments of $0.9 \mathrm{~m} / \mathrm{s}(2 \mathrm{mph})$ until the prototype started rotating. Then the wind speed was increased incrementally $2.2 \mathrm{~m} / \mathrm{s}$ ( $5 \mathrm{mph}$ ) from 11.2 up to $22.4 \mathrm{~m} / \mathrm{s}$ ( $25 \mathrm{up}$ to $50 \mathrm{mph})$. At each step, the revolution per minute (RPM), output voltage, and current were recorded.

The performance properties in the wind turbine tests, TSR and $C_{p}$ are determined by eqn (2) and eqn (3). The properties are compared between the GPT and scale HAWT prototypes in the experimental study.

$$
T S R=\frac{2 \mathrm{r} \pi \times \mathrm{RPM} / 60}{v}
$$


where

$\mathrm{r}=$ turbine radius $(\mathrm{m})$,

$\mathrm{RPM}=$ turbine rotation, revolution of per minute.

$$
C_{p}=\frac{P_{\text {out }}}{P_{\text {in }}}=\frac{V \times I}{P_{\text {in }}}
$$

where

$P_{\text {out }}=$ power output of turbine $(\mathrm{kW})$,

$V=$ electrical voltage generated by a wind turbine $(\mathrm{V})$,

$I=$ electrical current generated by a wind turbine (A).

\subsection{Test results and discussion}

The following test results and discussion are based on the propeller type C. As can be seen in Fig. 6(a), the TSR for the two HAWT prototypes are constantly lower than two and three levels of GPT. However, GPT-1 has lower TSR than HAWT-2. The increasing trend of TSR slows down at higher wind speeds. Fig. 6(b) compares the TSR for GPT-1 and the propeller of GPT installed horizontally (GPT-H). It can be observed that in lower wind speeds at less than $13.4 \mathrm{~m} / \mathrm{s}(30 \mathrm{mph})$, the increase of TSR is negligible. When the wind speed increases up to $22.4 \mathrm{~m} / \mathrm{s}(50 \mathrm{mph})$, the difference is about $100 \%$.

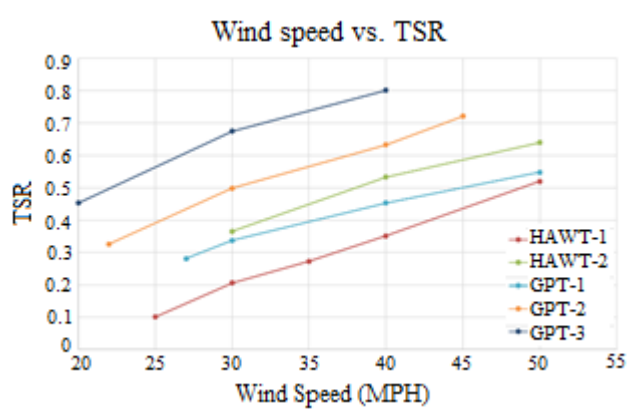

(a)

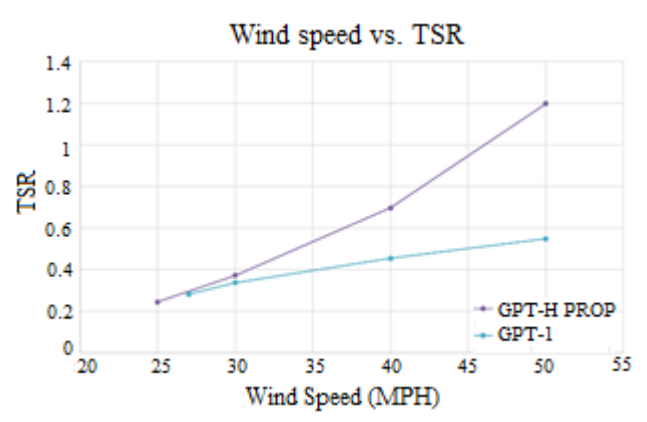

(b)

Figure 6: Comparison of GPT vs. HAWT and horizontal GPT propeller.

Fig. 7(a) shows the output power of the GPT and HAWT. It can be observed that GPT-2 output power can be up to about 200-250\% higher than HAWT-2. This is because GPT-2 has more swept area. However, GPT-1 has about 104\% higher power than HAWT-1 even though the swept area of GPT-1 is about $44 \%$ smaller than HAWT-1. It can be also observed that higher wind speed leads to a bigger gap between GPT and HAWT. Fig. 7(b) illustrates that, at low wind speeds, the performance of GPT in terms of power output is pretty close to that of the propeller of GPT installed horizontally $(\mathrm{GPT}-\mathrm{H})$. This indicates the negligible impact of GPT's structure on power loss at low wind speeds.

Fig. 8(a) compares GPT with HAWTs in terms of power coefficient $\left(C_{p}\right)$. It can be observed that increasing the layers has led to an increase in power output, which is constantly higher than that of HAWTs prototypes. There is an irregular jump in GPT-1 for low wind 


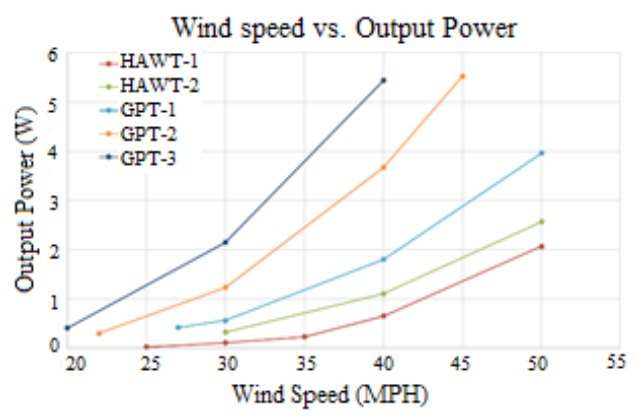

(a)

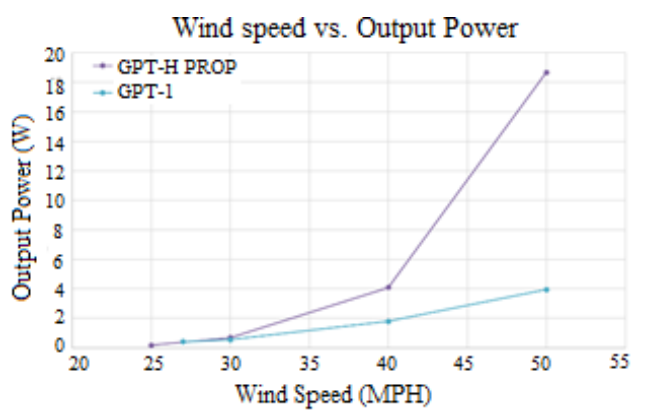

(b)

Figure 7: Power output comparison of GPT vs. HAWT and horizontal GPT propeller.

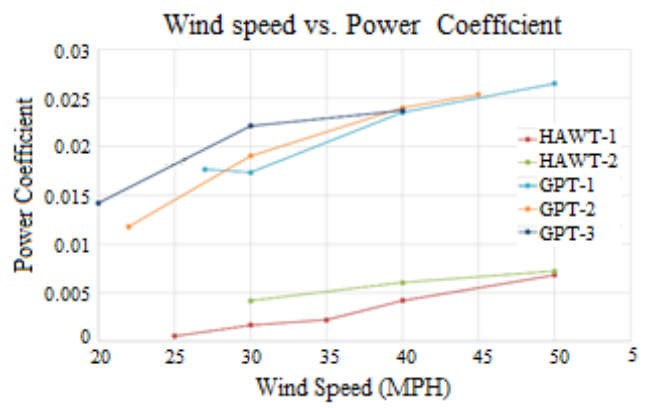

(a)

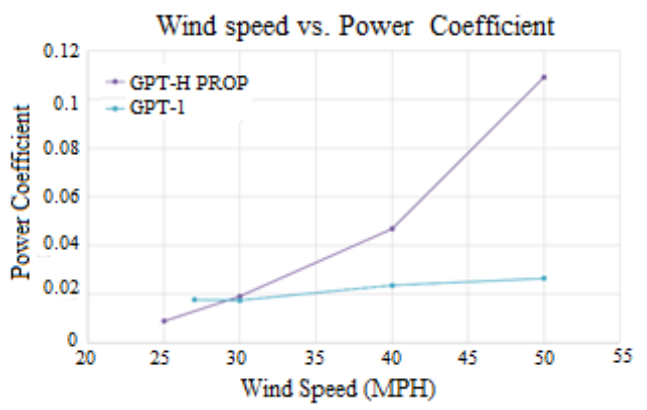

(b)

Figure 8: $C_{p}$ comparison of GPT vs. HAWTs and horizontal GPT propeller.

speed, which could be explained by the different cut-off speed of the system when there is only one level of GPT. Fig. 8(b) indicates that, at low wind speed, GPT-1 can have a better performance comparing to that of the propeller of GPT installed horizontally (GPT-H), although, at higher wind speeds, it shows a negative impact.

\section{APPLICATION PERSPECTIVE OF THE GPT}

The GPT has all advantages and characteristics of the VAWTs. In addition, the GPT does not have exposed rotating parts. All rotating parts are covered in enclosures. Therefore, the GPT is safer and more environment friendly than other VAWTs. The GPT is modularstackable. One module is one level of the GPT including one enclosure and one turbine. Therefore, each level of the GPT can be prefabricated in factory and assembled at site. The modules are stackable in multiple levels to meet the designed power. At the installation site, only light work is required. The GPT can have many applications, for instance, on buildings, towers, and bridges, including wind farms. Some of these potential applications are illustrated in Fig. 9.

\section{CONCLUSION}

The unique modular VAWT called GPT has a cylindrical shape which is able to generate electricity from $360^{\circ}$ wind flow. Turbine blades and shaft of the GPT are entirely covered in 


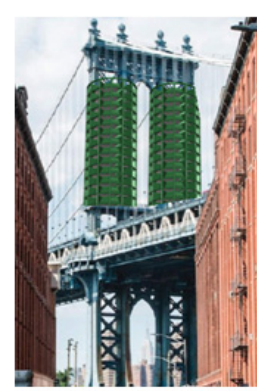

Bridge
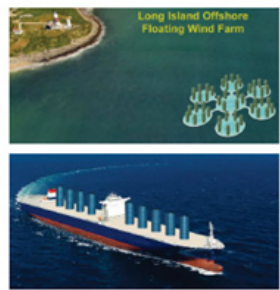

Wind Farm

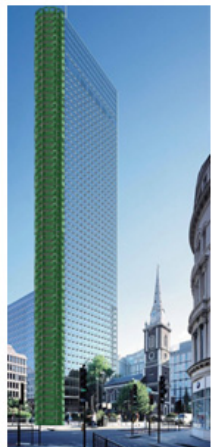

Building

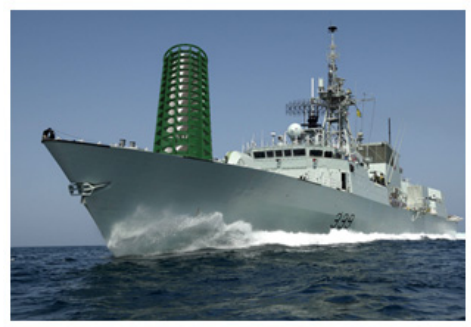

Ship

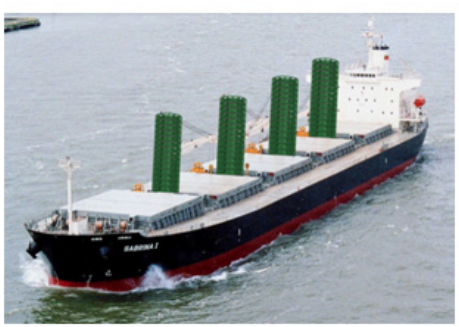

Vessel
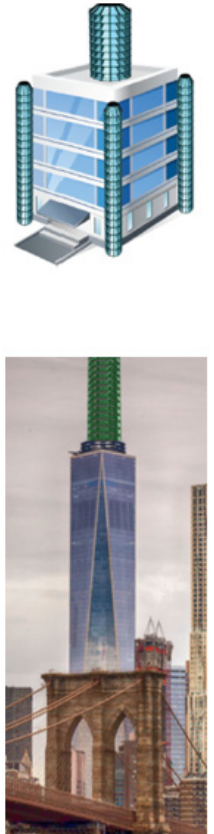

Tower

Figure 9: Potential Applications of the GPT.

a round enclosure. No moving components are exposed to outside. The GPT is quiet, safe, and environment friendly. In operation, the wind stream alternates the flow direction in the enclosure by the redirecting bottom wall and blows toward the turbine blades vertically. In addition, the modular and packable characteristics of the GPT make its installation convenient to meet the required power generation capacity by using multiple levels. To evaluate the performance of the GPT, scaled GPTs were designed and tested in the wind tunnel. The performance of the GPT was compared with equivalent scale HAWT prototypes. The test results showed that the GPT could produce more power compared to the HAWTs in the study. The $C_{p}$ was also constantly higher than HAWTs, which indicates that the GPT with more levels has the potential to be competitive with, at least, small or medium-sized HAWTs. Some of the observations in the study are summarized as follows:

- A higher number of levels in GPT leads to higher power generation since more levels will have greater swept area. This increase is not linear, and the gap further increases in higher wind speeds.

- The increase in power coefficient slows down in higher wind speed. When wind speed is up to $13.4 \mathrm{~m} / \mathrm{s}(30 \mathrm{mph})$, the increase is significant.

- In all prototypes, the cut-off speed for GPT is smaller than HAWT prototypes by about $15-40 \%$ depending on the swept area.

- The relation between the number of levels in GPT and power generation is closer to being linear in low wind speed up to $13.4 \mathrm{~m} / \mathrm{s}(30 \mathrm{mph})$. 
In light of the modular and stackable characteristics of the GPT, the manufacturing and installation of the GPT are economically justified. The operation of the GPT is safe and environment friendly. The performance of the actual size GPT will be further studied.

\section{REFERENCES}

[1] Clancy, K. Grumke, K. \& Toombs, Z., Wind energy is booming in Middle America and creating a huge demand for workers who can keep turbines spinning. https://www.newsy.com/stories/wind-turbine-technician-is-fastest-growing-job-inus/. Accessed on: 13 Sep. 2021.

[2] Möllerström, E., Ottermo, F., Goude, A., Eriksson, S., Hylander, J. \& Bernhoff, H., Turbulence influence on wind energy extraction for a medium size vertical axis wind turbine. Wind Energy, 19(11), pp. 1963-1973, 2016.

[3] Kjellin, J., Eriksson, S. \& Bernhoff, H., Electric control substituting pitch control for large wind turbines. Journal of Wind Energy, 2013, 342061, 2013.

[4] Dalili, A.E.R.N., A review of surface engineering issues critical to wind turbine performance. Renewable and Sustainable Energy Reviews, 13(2), pp. 428-438, 2009.

[5] Österberg, D., Multi-body unsteady aerodynamics in 2D applied to a vertical-axis wind turbine using a vortex method, Uppsala Universitet, Uppsala, Sweden, 2010.

[6] Hui, I., Cain, B. \& Dabiri, J., Public receptiveness of vertical axis wind turbines. Energy Policy, 112, pp. 258-271, 2018.

[7] Drake, D., Schumacher, S. \& Sponsler, M., Regional analysis of wind turbine-caused bat and bird fatality, Environmental and economic research and development program, Wisconsin's Focus on Energy, 2012.

[8] Smallwood, K.S. \& Karas, B., Avian and bat fatality rates at old-generation and repowered wind turbines in California. The Journal of Wildlife Management, 73(7), pp. 1062-1071, 2009.

[9] Thomas, R.N., Coupled vortex vertical axis wind turbine. United States of America Patent US6784566B2, 31 Aug. 2004.

[10] Reddy, K.A. \& Dagamoori, K., A brief research study, design and analysis on wind turbine. Journal of Modern Engineering Research, 5(10), pp. 5-30, 2015.

[11] Óskarsdóttir, M.Ó., A general description and comparison of horizontal axis wind turbines and vertical axis wind turbines, University of Iceland, Reykjavik, 2014.

[12] Omijeh, B.O., Nmom, C.S. \& Nlewem, E., Modeling of a vertical axis wind turbine with permanent magnet synchronous generator for Nigeria. International Journal of Engineering and Technology, 3(2), pp. 212-220, 2013. 